\title{
Chalcogenide Perovskites - an Emerging Class of Ionic Semiconductors
}

Samanthe Perera ${ }^{\mathrm{a}, 1}$, Haolei Hui ${ }^{\mathrm{a}, \mathrm{b}, 1}$, Chuan Zhao ${ }^{\mathrm{a}}$, Hongtao Xue ${ }^{\mathrm{a}}$, Fan Sun ${ }^{\mathrm{a}}$, Chenhua Deng ${ }^{\mathrm{c}}$,

Nelson Gross ${ }^{\mathrm{a}}$, Chris Milleville ${ }^{\mathrm{d}}$, Xiaohong Xu ${ }^{\mathrm{c}}$, David F. Watson ${ }^{\mathrm{d}}$, Bernard Weinstein ${ }^{\mathrm{a}}$, YiYang Sun ${ }^{\mathrm{e},}$, Shengbai Zhang ${ }^{\mathrm{e}}$ and Hao Zeng ${ }^{\mathrm{a},{ }^{*}}$

${ }^{\mathrm{a}}$ Department of Physics, University at Buffalo, the State University of New York, Buffalo NY 14260, USA

${ }^{b}$ School of Science, Xi'an Jiaotong University, Xi'an, 710049, China

${ }^{c}$ Key Laboratory of Magnetic Molecules and Magnetic Information Material of Ministry of Education, School of Chemistry and Materials Science of Shanxi Normal University,

\section{Linfen 041004, China}

${ }^{d}$ Department of Chemistry, University at Buffalo, the State University of New York, Buffalo, NY 14260, USA

${ }^{e}$ Department of Physics, Applied Physics \& Astronomy, Rensselaer Polytechnic Institute, Troy, New York 12180, USA

${ }^{1}$ These authors contributed equally to this work.

* Corresponding Authors: suny4@rpi.edu, haozeng@buffalo.edu 


\begin{abstract}
We report the synthesis and characterization of a novel class of ionic semiconductor materials inorganic chalcogenide perovskites. Several different compounds including $\mathrm{BaZrS}_{3}, \mathrm{CaZrS}_{3}$, $\mathrm{SrTiS}_{3}$ and $\mathrm{SrZrS}_{3}$ were synthesized by high temperature sulfurization of their oxide counterparts. Their crystal structures were identified by XRD and composition by EDX. UV-vis and photoluminescence measurements confirmed that they are direct gap semiconductors with band gap values consistent with theoretical predictions. By adopting an anion alloying approach, we demonstrate widely tunable band gap from $1.73 \mathrm{eV}$ to $2.87 \mathrm{eV}$. These strongly ionic semiconductors provide a new avenue for engineering the semiconducting properties for applications such as energy harvesting, solid state lighting and sensing.
\end{abstract}

KEYWORDS: chalcogenide perovskite, sulfurization, band gap engineering, ionic semiconductor 
Semiconductor materials have wide ranging industrial applications from microelectronics to energy harvesting and sensing. Conventional semiconductors that dominate the current industry are mainly covalent materials, as characterized by four-fold coordination of all atoms. Recently, the photovoltaic research community has focused their attention on an unconventional material: the organic-inorganic halide perovskite. The power conversion efficiency (PCE) of perovskite solar cells has experienced phenomenal increase from an initial value of $3.8 \%$ in 2009 [1] to $15 \%$ in 2013 [2-5], and then quickly to $21.0 \%$ in another two years [6-8][9]. Perovskites, however, are ionic materials as characterized by higher coordination maximizing the Coulomb interaction between cations and anions. It is likely that this ionicity could be a major contributing factor for the excellent transport properties of the halide perovskites, such as the extremely long carrier diffusion length [10-12]. It has been reported that in such materials deep level defects are less likely to form $[13,14]$. The great success of the halide perovskite materials has inspired us to search for novel semiconductor materials that can retain the excellent optoelectronic properties of halides, while avoiding their severe limitations such as instability and toxicity $[15,16]$.

As opposed to their oxide and halide siblings, chalcogenide perovskites have received little attention, despite being synthesized more than half a century ago [17, 18]. Publications on these materials are surprisingly scarce [19-21], and as a result, there is limited knowledge of their physical properties [22, 23]. Our recent theoretical study [24] have screened $18 \mathrm{ABX}_{3}$ chalcogenide materials $(\mathrm{A}=\mathrm{Ca}, \mathrm{Sr}$, or $\mathrm{Ba} ; \mathrm{B}=\mathrm{Ti}, \mathrm{Zr}$, or $\mathrm{Hf} ; \mathrm{X}=\mathrm{S}$ or $\mathrm{Se})$ with several exciting discoveries: 1) These materials in a distorted perovskite structure are semiconductors with band gaps ranging from 0.3 to $2.3 \mathrm{eV}$; 2) They could possess direct band gaps with high optical absorption coefficients, comparable to those of traditional optoelectronic semiconductors such as GaAs; 3) The band dispersion is large for those $\mathrm{Zr}$ and $\mathrm{Hf}$ compounds, suggesting good carrier 
mobility. With these predicted properties, these materials are particularly attractive for photovoltaic and optoelectronic applications and therefore call for experimental verification.

In this paper, we report the synthesis of polycrystalline chalcogenide perovskites $\left(\mathrm{BaZrS}_{3}\right.$, $\mathrm{CaZrS}_{3}, \mathrm{SrTiS}_{3}$ and $\mathrm{SrZrS}_{3}$ ) and the characterization of their structural and physical properties. These materials were synthesized by sulfurization of oxide perovskites by $\mathrm{CS}_{2}$ at high temperatures. X-ray diffraction (XRD) shows that $\mathrm{BaZrS}_{3}$ possesses a distorted perovskite structure. Low temperature Raman measurements reveal multiple modes ranging from 50 to 400 $\mathrm{cm}^{-1}$, in good agreement with theoretical predictions. UV-vis absorption spectroscopy confirms that it is a semiconductor, with high absorbance from UV to visible region. The band gap value is estimated to be $1.73 \mathrm{eV}$, again in good agreement with theoretical predictions. The sample also show photoluminescence (PL) in the visible region, confirming its direct band gap. This material shows excellent stability in ambient conditions as compared to halide perovskites. We further demonstrate systematic band gap engineering by anion alloying in oxysulfides.

\section{Experimental}

Synthesis of $\mathrm{BaZrS}_{3}$. A ceramic boat containing $0.6 \mathrm{~g}$ of $\mathrm{BaZrO}_{3}(\geq 99 \%$ purity, Alfa Aesar) was heated at a rate of $15^{\circ} \mathrm{C} / \mathrm{min}$ in a $2 "$ quartz tube furnace under flowing $\mathrm{Ar}$. $\mathrm{CS}_{2}(\geq 99.8 \%$ purity, Alfa Aesar) was introduced at $800{ }^{\circ} \mathrm{C}$ by bubbling Ar through a gas bubbler containing $\mathrm{CS}_{2}$. Once the temperature reached $1050{ }^{\circ} \mathrm{C}$, it was kept constant for 4 hours before cooling down to $800{ }^{\circ} \mathrm{C}$ at the same rate. At $800{ }^{\circ} \mathrm{C}, \mathrm{CS}_{2}$ flow was turned off and the temperature was kept constant for another 2 hours under Ar flow to remove unreacted sulfur, before cooling down to room temperature. The exhaust gas from the tube was bubbled through $1 \mathrm{M} \mathrm{NaOH}$ solution throughout the reaction to remove excess $\mathrm{CS}_{2}$. The $\mathrm{CS}_{2}$ flow rate was adjusted from 10 to 20 
standard cubic centimeters per minute ( $\mathrm{sccm}$ ). The reaction temperature and time were optimized to obtain phase pure $\mathrm{BaZrS}_{3}$ without oxides or other impurity phases.

Synthesis of oxysulfides. Seven $\mathrm{BaZrO}_{3}$ powder samples were placed in the uniform temperature region in a two zone furnace at an interval of $1 \mathrm{~cm}$ apart inside the quartz tube. The sample closest to upstream is labeled as S1 and the one farthest is labeled as S7. The synthesis was carried out similar to that of $\mathrm{BaZrS}_{3}$. The flow rate of $\mathrm{CS}_{2}$ was kept at $15 \mathrm{sccm}$. By limiting the $\mathrm{CS}_{2}$ flow and controlling the position of the $\mathrm{BaZrO}_{3}$ powder, sulfur deficiency was enforced in these samples. $\mathrm{BaZrO}_{3}$ located the closest to the upstream (S1) was completely sulfurized, while S7 remains nearly un-sulfurized; in between, oxysulfides $B a Z r\left(O_{x} S_{1-x}\right)_{3}$ with various degree of sulfurization were achieved.

Crystal structure and phase information was acquired using a Rigaku Ultima IV X-ray diffraction (XRD) system operating at $1.76 \mathrm{~kW}(40 \mathrm{kV}, 44 \mathrm{~mA})$ with $\mathrm{Cu}$ target for X-ray source and dual position graphite diffracted beam monochromator.

UV-vis absorption spectra were obtained by measuring diffuse reflectance with a wavelength ranged from $400 \mathrm{~nm}$ to $1100 \mathrm{~nm}$ using Labsphere RSA-HP-8453 reflectance spectroscopy accessory attached to Agilent 8453 ultra-violet/visible (UV-vis) spectroscopy system.

Raman and PL measurements Stokes Raman was collected using RCA photomutiplier tube attached to a Jobin Yvonne U1000 double monochromator with 1800 lines/mm holographic gratings. For excitation 4-5 mw of the $676.457 \mathrm{~nm}$ line from a Coherent Innova $100 \mathrm{Kr}^{+}$ion laser was focused with a $300 \mathrm{~mm}$ lens to produce a spot size of $\sim 130 \mu \mathrm{m}$. Spectra were averaged three times and smoothed with 5 point adjacent averaging. 
Morphology and energy-dispersive X-ray elemental analysis was carried out using a Hitachi SU70 field emission scanning electron microscope (FESEM) with Oxford energy-dispersive Xray spectrometer (EDS) running a silicon drift detector.

First-principles calculation was based on density functional theory using the PBEsol functional [25] to optimize the lattice constants and atomic coordinates. Band structure was calculated using the HSE06 hybrid functional [26]. Raman spectrum calculations (frequency and intensity) were carried out following Ref. [27] using the PBEsol functional.

\section{Results and discussions}

Typical SEM images of the polycrystalline $\mathrm{BaZrO}_{3}$ precursor and $\mathrm{BaZrS}_{3}$ powder sample are shown in Fig. 1(a) and 1(b), respectively. The $\mathrm{BaZrO}_{3}$ precursor has a grain size of $100-200 \mathrm{~nm}$. After sulfurization, the particles become much larger of a few $\mu \mathrm{m}$ in size. The particles are faceted suggesting good crystallinity. The color of $\mathrm{BaZrO}_{3}$ is white (inset of Fig. 1(a)), suggesting no optical absorption at the visible range, consistent with its band gap value of $5 \mathrm{eV}$. After sulfurization, the sample becomes black (inset of Fig. 1(b)), suggesting visible light absorption in a broad range. EDX analysis (Fig. 1(c)) shows a composition close to stoichiometry, with the atomic ratios of Ba: $\mathrm{Zr}: \mathrm{S}=21.6: 21.4$ : 57.0. The slight off-stoichiometry from $\mathrm{ABX}_{3}$ is not uncommon in perovskites, and mainly reflects the presence of sulfur vacancies due to high temperature treatment. The XRD spectrum of $\mathrm{BaZrS}_{3}$ is shown in Fig. 2. It can be seen that all peaks can be matched to those of the standard file JCPDS 00-015-0327. This confirms that the sample possesses an orthorhombic distorted perovskite structure with Pnma space group. The lattice constants are measured to be $a=7.04 \AA, b=9.98 \AA$ and $c=7.05 \AA$, respectively, closely matched to the published results [18]. No secondary phase is detected within the instrument limit. 
Raman spectra measured at different temperatures from 14 to $295 \mathrm{~K}$ are shown in Fig. 3(a). At room temperature, roughly 5 broad peaks can be observed. With decreasing temperature, these 5 peaks are progressively sharpened due to the customary decrease in anharmonic thermal phonon-decay. As a result, the broad peak centered at about $215 \mathrm{~cm}^{-1}$ is shown to consist of two peaks at 216 and $224 \mathrm{~cm}^{-1}$, which are identified to be $A_{\mathrm{g}}{ }^{6}$ and $B_{2 \mathrm{~g}}{ }^{6}$ modes, respectively. At the reduced measuring temperatures of $14-150 \mathrm{~K}$, the sharpening concentrates the mode oscillator strengths into enhanced peak heights permitting weaker additional features to be detected, e.g., at $169 \mathrm{~cm}^{-1}$ and $304 \mathrm{~cm}^{-1}$. The low temperature Raman measurements allow direct comparison with first-principles calculations. According to group theory, orthorhombic Pnma structure should have 24 Raman modes, $7 A_{\mathrm{g}}+5 B_{1 \mathrm{~g}}+7 B_{2 \mathrm{~g}}+5 B_{3 \mathrm{~g}}$ [28-30]. The calculated peak position and intensity are shown in Fig. 3(b), together with the experimental spectrum measured at $14 \mathrm{~K}$. In total, 14 Raman modes can be identified with theoretical assignments given in Table 1 . The peak positions show good agreement with experimental data. On the other hand, as seen from Fig. 3(c), $\mathrm{BaZrO}_{3}$ shows no Raman peak at this frequency range, which is expected since $\mathrm{BaZrO}_{3}$ is cubic (space group Pm3m) with no significant first-order Raman scattering [31]. The Raman results, combined with $\mathrm{XRD}$ results, show that our $\mathrm{BaZrS}_{3}$ is phase pure with good crystalline quality.

Table 1. Assignment of experimental Raman peaks according to the irreducible representations of point group $D_{2 \mathrm{~h}}$ based on calculated frequencies. The unit is $\mathrm{cm}^{-1}$.

\begin{tabular}{|l|l|l|l|l|l|l|l|l|}
\hline mode & Theory & Exp. & mode & Theory & Exp. & mode & Theory & Exp. \\
\hline$B_{3 \mathrm{~g}}{ }^{5}$ & 418.1 & -- & $B_{3 \mathrm{~g}}{ }^{3}$ & 203.0 & 207 & $A_{\mathrm{g}}{ }^{3}$ & 98.8 & 97 \\
\hline$B_{2 \mathrm{~g}}{ }^{7}$ & 399.1 & -- & $B_{2 \mathrm{~g}}{ }^{5}$ & 201.9 & 207 & $B_{2 \mathrm{~g}}{ }^{3}$ & 97.6 & 93 \\
\hline
\end{tabular}




\begin{tabular}{|l|l|l|l|l|l|l|l|l|}
\hline$B_{1 \mathrm{~g}}{ }^{5}$ & 388.0 & 392 & $B_{1 \mathrm{~g}}{ }^{3}$ & 174.5 & - & $\mathrm{B}_{1 \mathrm{~g}}{ }^{1}$ & 85.8 & 83 \\
\hline$A_{\mathrm{g}}{ }^{7}$ & 304.6 & 304 & $A_{\mathrm{g}}{ }^{5}$ & 168.7 & 169 & $B_{2 \mathrm{~g}}{ }^{2}$ & 81.3 & 78 \\
\hline$B_{1 \mathrm{~g}}{ }^{4}$ & 290.8 & -- & $B_{2 \mathrm{~g}}{ }^{4}$ & 167.8 & 169 & $B_{3 \mathrm{~g}}{ }^{1}$ & 76.9 & 72 \\
\hline$B_{3 \mathrm{~g}}{ }^{4}$ & 290.3 & -- & $B_{3 \mathrm{~g}}{ }^{2}$ & 160.2 & -- & $A_{\mathrm{g}}{ }^{2}$ & 76.0 & 72 \\
\hline$B_{2 \mathrm{~g}}{ }^{6}$ & 214.8 & 224 & $A_{\mathrm{g}}{ }^{4}$ & 142.7 & 143 & $B_{2 \mathrm{~g}}{ }^{1}$ & 64.0 & 64 \\
\hline$A_{\mathrm{g}}{ }^{6}$ & 207.0 & 216 & $B_{1 \mathrm{~g}}{ }^{2}$ & 111.0 & -- & $A_{\mathrm{g}}{ }^{1}$ & 58.7 & 57 \\
\hline
\end{tabular}

Figure 4 shows the band structure of $\mathrm{BaZrS}_{3}$ calculated using the HSE06 hybrid functional, which suggests that $\mathrm{BaZrS}_{3}$ is a direct band gap semiconductor with both conduction band minimum and valence band maximum located at the $\Gamma$ point. The band gap is $1.74 \mathrm{eV}$. It can also be seen that the bottom of conduction band has relatively large dispersion, hence good electron transport, even though it is mainly derived from the transition metal element $\mathrm{Zr}$. To verify the electronic structure calculations, UV-vis spectroscopy and PL have been performed. As can be seen from the UV-vis absorption spectrum in Fig. 5, $\mathrm{BaZrS}_{3}$ has an absorption tail extending to low energies, making accurate determination of the band gap difficult [32]. The band gap value is estimated to be about $1.73 \mathrm{eV}$, in good agreement with the theoretical prediction. As can be seen from the PL spectrum plotted in the same graph, a broad PL peak centered at about $1.7 \mathrm{eV}$ can be observed. The emission near the band gap energy confirms that $\mathrm{BaZrS}_{3}$ is a direct band gap material. The absorption and PL results suggest that $\mathrm{BaZrS}_{3}$ has desirable optical properties for energy harvesting and other optoelectronic applications.

We further demonstrate band gap engineering by anion alloying. Since $\mathrm{BaZrO}_{3}$ is an insulator with a band gap of about $5 \mathrm{eV}$ while that of $\mathrm{BaZrS}_{3}$ is about $1.8 \mathrm{eV}$. We postulate that the band gap can be systematically tuned by formation of oxysulfides with different $\mathrm{O}^{2-}$ to $\mathrm{S}^{2-}$ 
ratio. To achieve this, we intentionally tuned the chemical potential to favor the formation of oxysulfides, by limiting the flow of $\mathrm{CS}_{2}$ to ensure sulfur deficiency, while keeping the temperature at $1,050{ }^{\circ} \mathrm{C}$ to allow thermodynamically stable phase to be formed. With such an approach, the composition of the chalcogenide perovskites can be symmetrically tuned from essentially oxides $\left(\mathrm{BaZrO}_{3}\right)$ to nearly $100 \%$ sulfides $\left(\mathrm{BaZrS}_{3}\right)$. As can be seen from the EDX analysis (Table 2), the composition of $\mathrm{S}$ and $\mathrm{O}$ shows opposite trends from $\mathrm{S} 1$ to S7: while $\mathrm{S}$ increases from $3.4 \%$ for $\mathrm{S} 1$ to $55.8 \%$ for $\mathrm{S} 7$, O decreases from $51 \%$ to close to 0 , respectively.

Table 2. The atomic percentage of the 4 elements ( $\mathrm{Ba}, \mathrm{Zr}, \mathrm{O}$ and $\mathrm{S}$ ) for samples $\mathrm{S} 1$ to $\mathrm{S} 7$, as measured by EDX spectroscopy.

\begin{tabular}{|c|r|r|r|r|r|r|r|}
\hline & \multicolumn{1}{|c|}{ S1 } & \multicolumn{1}{c|}{ S2 } & \multicolumn{1}{c|}{ S3 } & \multicolumn{1}{c|}{ S4 } & \multicolumn{1}{c|}{ S5 } & \multicolumn{1}{c|}{ S6 } & S7 \\
\hline $\mathbf{B a}$ & 25.08 & 25.09 & 23.42 & 21.98 & 25.54 & 25.64 & 23.79 \\
\hline $\mathbf{Z r}$ & 20.68 & 20.9 & 21.26 & 20.31 & 21.48 & 22.23 & 22.31 \\
\hline $\mathbf{O}$ & 50.85 & 46.55 & 37.1 & 33.68 & 23.59 & 19.74 & 0 \\
\hline $\mathbf{S}$ & 3.38 & 7.46 & 18.22 & 24.03 & 29.4 & 32.4 & 53.89 \\
\hline
\end{tabular}

The color of the samples from S1 to S7 changes progressively from nearly white to completely black, as shown by the optical images of these samples in Fig. 6(a). UV-vis absorption spectra (Fig. 6(b)) show that with increasing S content, the optical absorption progressively red-shifts to longer wavelengths (lower energies). The estimated band gap values are plotted as a function of $\mathrm{S}$ concentration in Fig. 6(c). It is seen that $E_{\mathrm{g}}$ decreases monotonically from $2.87 \mathrm{eV}$ for $\mathrm{S} 1$ to $1.75 \mathrm{eV}$ for $\mathrm{S} 7$. It is straightforward to understand the 
tuning of the band gap: since the valence band of these perovskites consists of mainly the p states of $\mathrm{O} / \mathrm{S}$ anions, and $\mathrm{O} 3 p$ states are deeper in energy than that of $\mathrm{O} 2 p$ states, replacing $\mathrm{O}^{2-}$ with $\mathrm{S}^{2-}$ effectively narrows the band gap by uplifting the valence band minimum (VBM). Nevertheless, it is fascinating that the band gap can be systematically tuned over a wide range by the degree of sulfurization. Such range is of great interest to broad applications from energy harvesting, solid state lighting to sensing.

Other chalcogenide perovskites including $\mathrm{CaZrS}_{3}, \mathrm{SrZrS}_{3}$ and $\mathrm{SrTiS}_{3}$ have also been synthesized using similar techniques. $\mathrm{CaZrS}_{3}$ is found to be in distorted perovskite structure with a band gap of $1.90 \mathrm{eV}$ (Fig. 6(b)), while both $\mathrm{SrTiS}_{3}$ and $\mathrm{SrZrS}_{3}$ possess a needle-like phase. Both are predicted to have very low band gap values beyond the range of our UV-vis spectrometer. The stability of $\mathrm{BaZrS}_{3}$ was tested by measuring the XRD pattern and UV-vis absorption spectrum of a sample stored in air for 7 months. No detectable change of properties has been observed (Fig. S3). Repeated washing of $\mathrm{BaZrS}_{3}$ by deionized water also does not result in change of its properties.

In conclusion, several chalcogenide and oxysulfide perovskite compounds including $\mathrm{BaZrS}_{3}$, $\mathrm{CaZrS}_{3}, \mathrm{SrZrS}_{3}, \mathrm{SrTiS}_{3}$ and $\mathrm{BaZr}\left(\mathrm{O}_{\mathrm{x}} \mathrm{S}_{1-\mathrm{x}}\right)_{3}$ have been synthesized. These materials belong to a new class of ionic semiconductors. The band gap of these materials can be systematically tuned in a wide range from UV to infrared. Due to their predicted strong iconicity they may exhibit unique physical properties such as free of deep level defects, which is beneficial for energy harvesting and other optoelectronic applications. It should be pointed out that oxide perovskites, with a chemical formula of $\mathrm{ABO}_{3}$, has long been an active field of research. This family of materials exhibits unusually rich properties ranging from colossal magnetoresistance, ferroelectricity to superconductivity and charge density waves, resulting from interplay of 
different degrees of freedoms with similar energy scales. The intriguing physics of their chalcogenide counterparts, however, is largely unexplored. Developing chalcogenide perovskite materials therefore not only provide new materials complementing oxides, but also offers new opportunities for exploring new physical phenomena and material properties tunable by light, electric and magnetic fields.

\section{Acknowledgement:}

Work supported by NSF DMR-1104994, CBET-1510121, and MRI-1229208. HLH supported by the Education Program for Talented Students of Xi'an Jiaotong University. 


\section{Figure captions:}

Figure 1. SEM images of the polycrystalline (a) $\mathrm{BaZrO}_{3}$ powder used for the synthesis and (b) the synthesized $\mathrm{BaZrS}_{3}$ powder sample; (c) the EDX spectrum of $\mathrm{BaZrS}_{3}$. Insets of Fig. 1(a) and (b) are the optical images of the white $\mathrm{BaZrO}_{3}$ powder and black $\mathrm{BaZrS}_{3}$ powder, respectively. The scale bars are $5 \mu \mathrm{m}$.

Figure 2. The XRD pattern of the $\mathrm{BaZrS}_{3}$. All peaks can be matched to the standard file JCPDS 00-015-0327 of the orthorhombic structured $\mathrm{BaZrS}_{3}$ (space group Pnma).

Figure 3. (a) The Raman spectra of $\mathrm{BaZrS}_{3}$ measured at different temperatures from 14 to $295 \mathrm{~K}$;

(b) the calculated Raman modes shown together with the spectrum measured at $14 \mathrm{~K}$; (c) Raman spectrum of $\mathrm{BaZrO}_{3}$.

Figure 4. Top view (a) and side view (b) of $\mathrm{BaZrS}_{3}$ in the distorted perovskite structure (space group Pnma). Red dashed frames and black arrows mark the unit cell and the crystallographic orientations, respectively. (c) Band structure of $\mathrm{BaZrS}_{3}$ calculated using the HSE06 hybrid functional. The high-symmetry $k$-points $\mathrm{X}, \mathrm{Y}, \mathrm{Z}, \mathrm{U}, \mathrm{S}, \mathrm{R}$, and $\Gamma$ correspond to $(\pi / a, 0,0),(0, \pi / b$, $0),(0,0, \pi / c),(\pi / a, 0, \pi / c),(\pi / a, \pi / b, 0),(\pi / a, \pi / b, \pi / c)$, and $(0,0,0)$, respectively, in the Brillouin zone. The rightmost panel shows the total density of states (DOS) and the site-projected DOS on Zr 4d and S 3p orbitals.

Figure 5. The UV-vis absorption spectrum and PL spectrum of $\mathrm{BaZrS}_{3}$.

Figure 6. (a) The optical images and (b) the UV-vis absorption spectra of the BaZr-oxysulfides with different degrees of sulfurization; and (c) the measured band gap energy as a function of sulfur atomic concentration. 


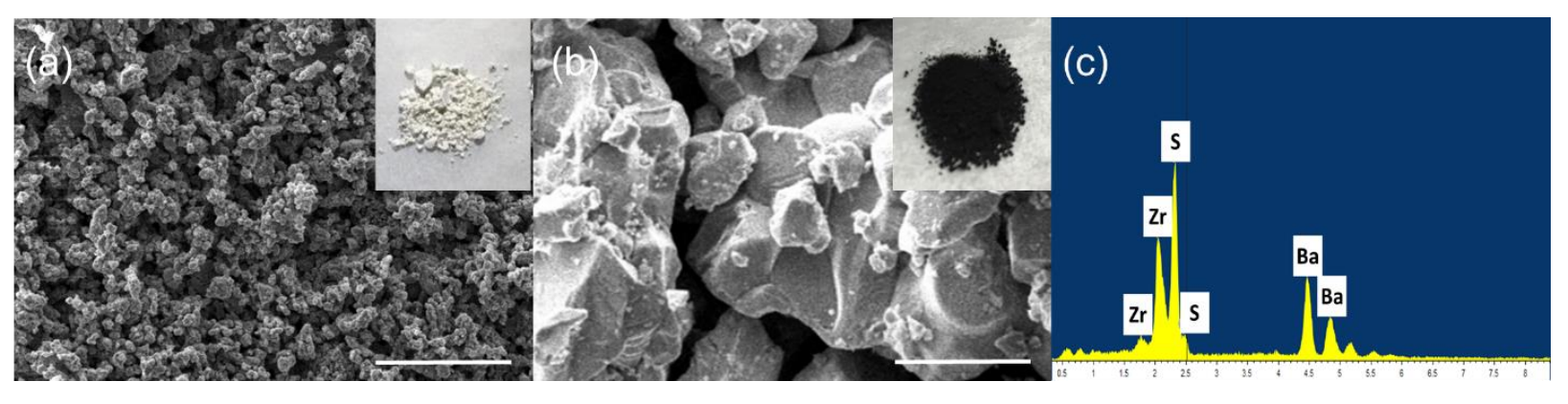

Figure 1 


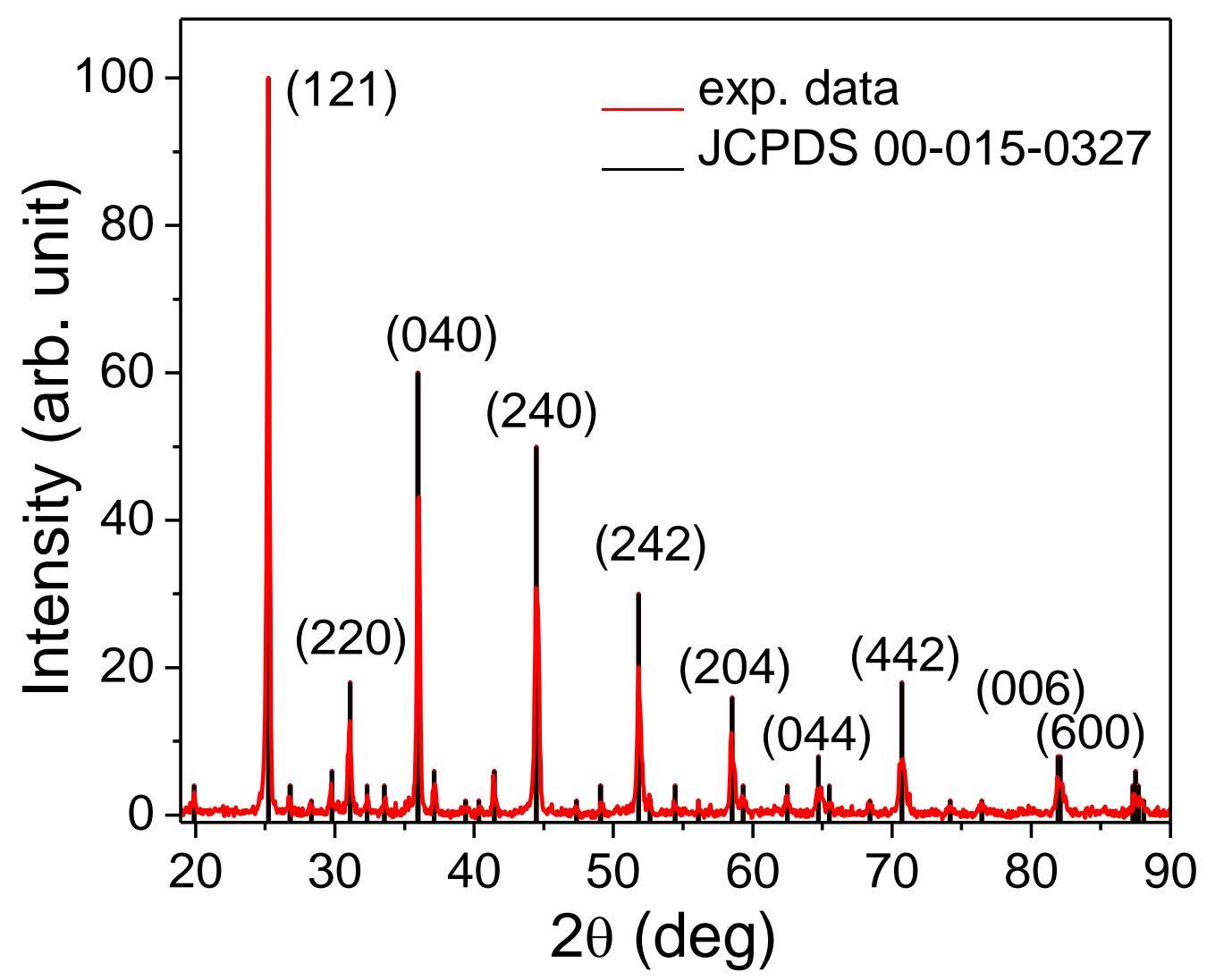

Figure 2 

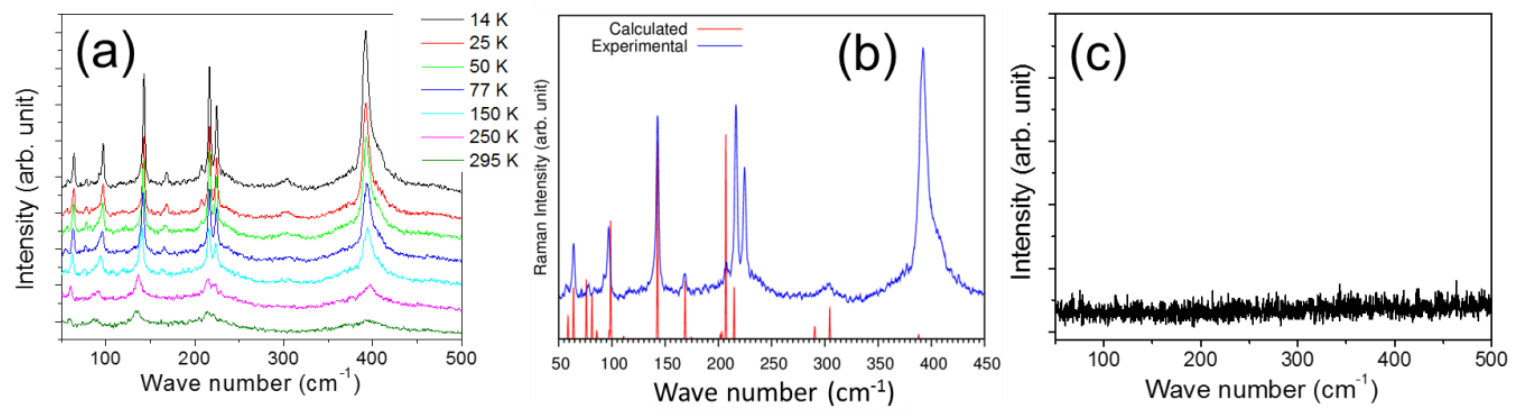

Figure 3 


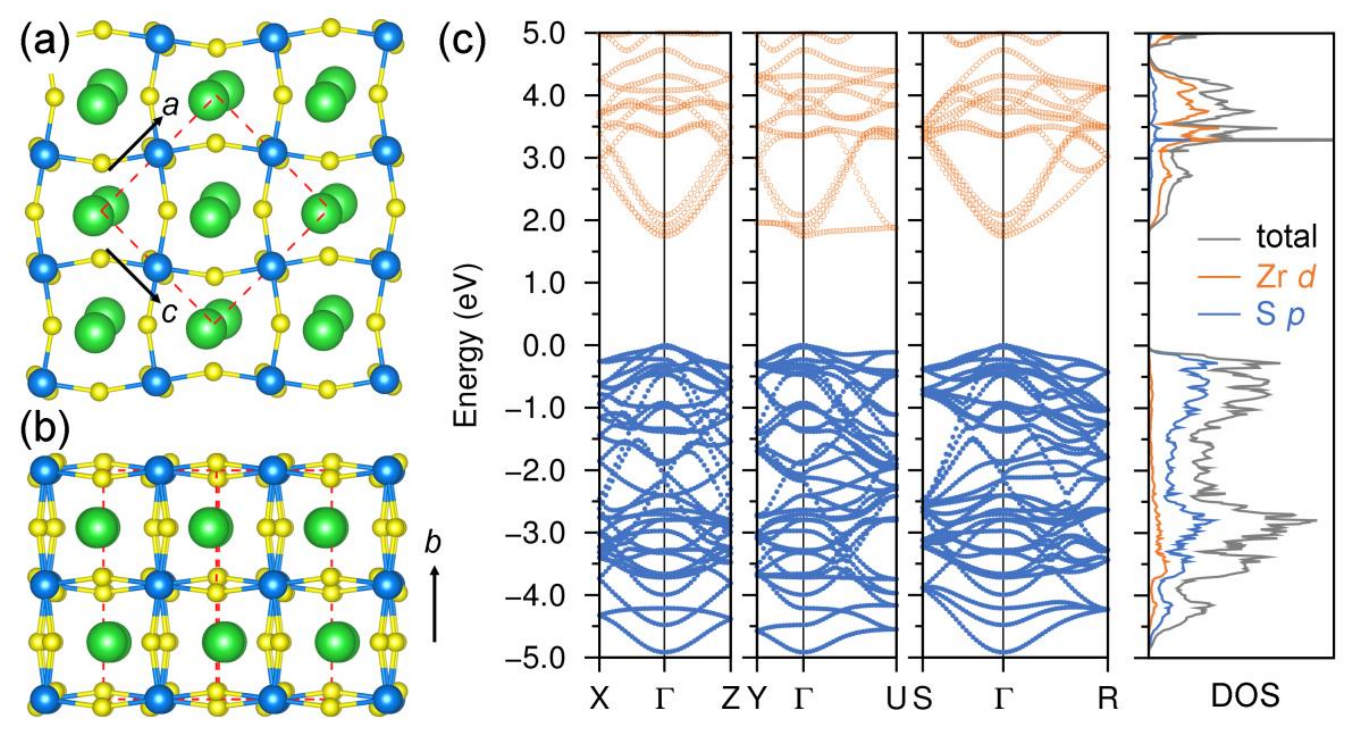

Figure 4 


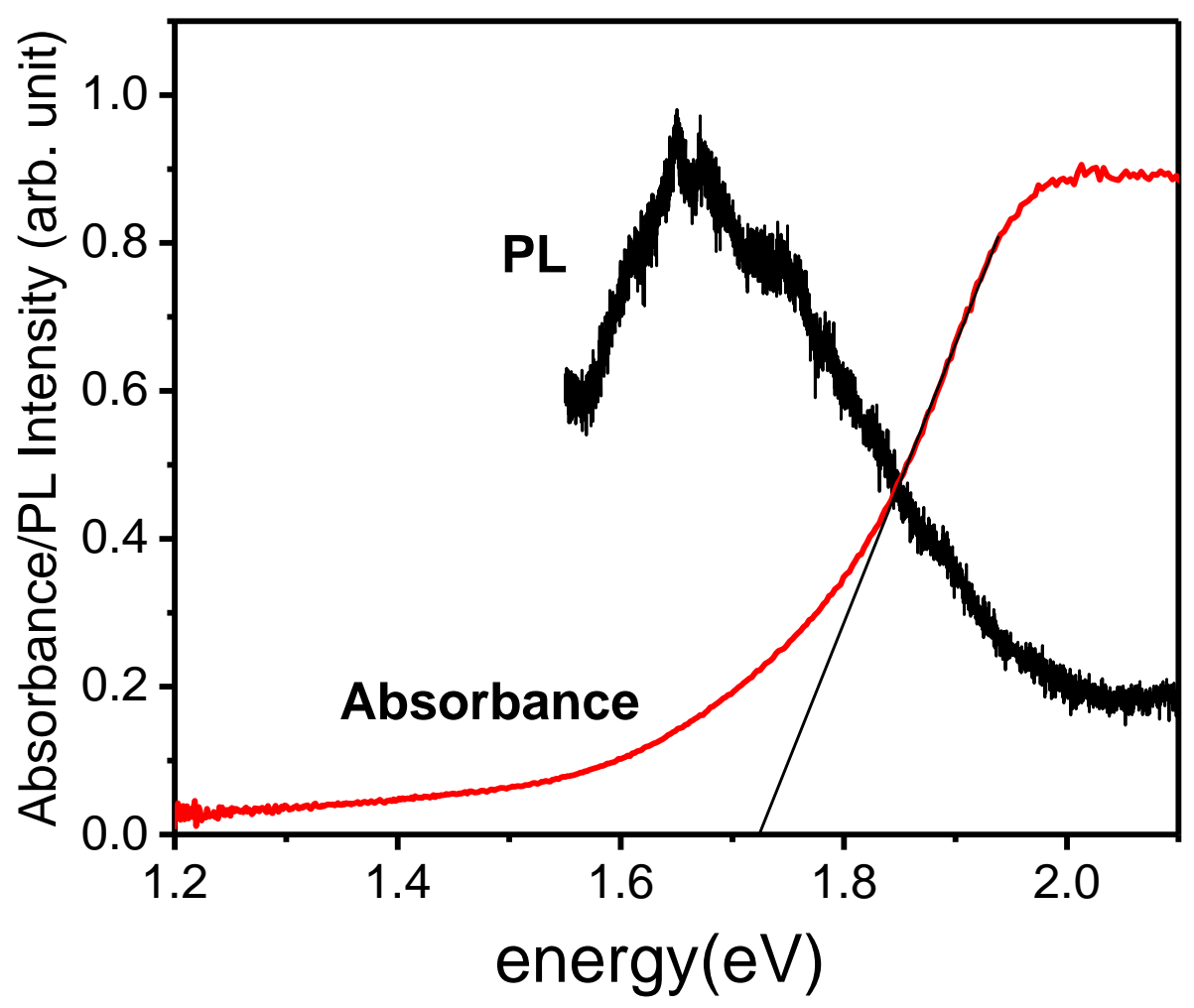

Figure 5 
(a)
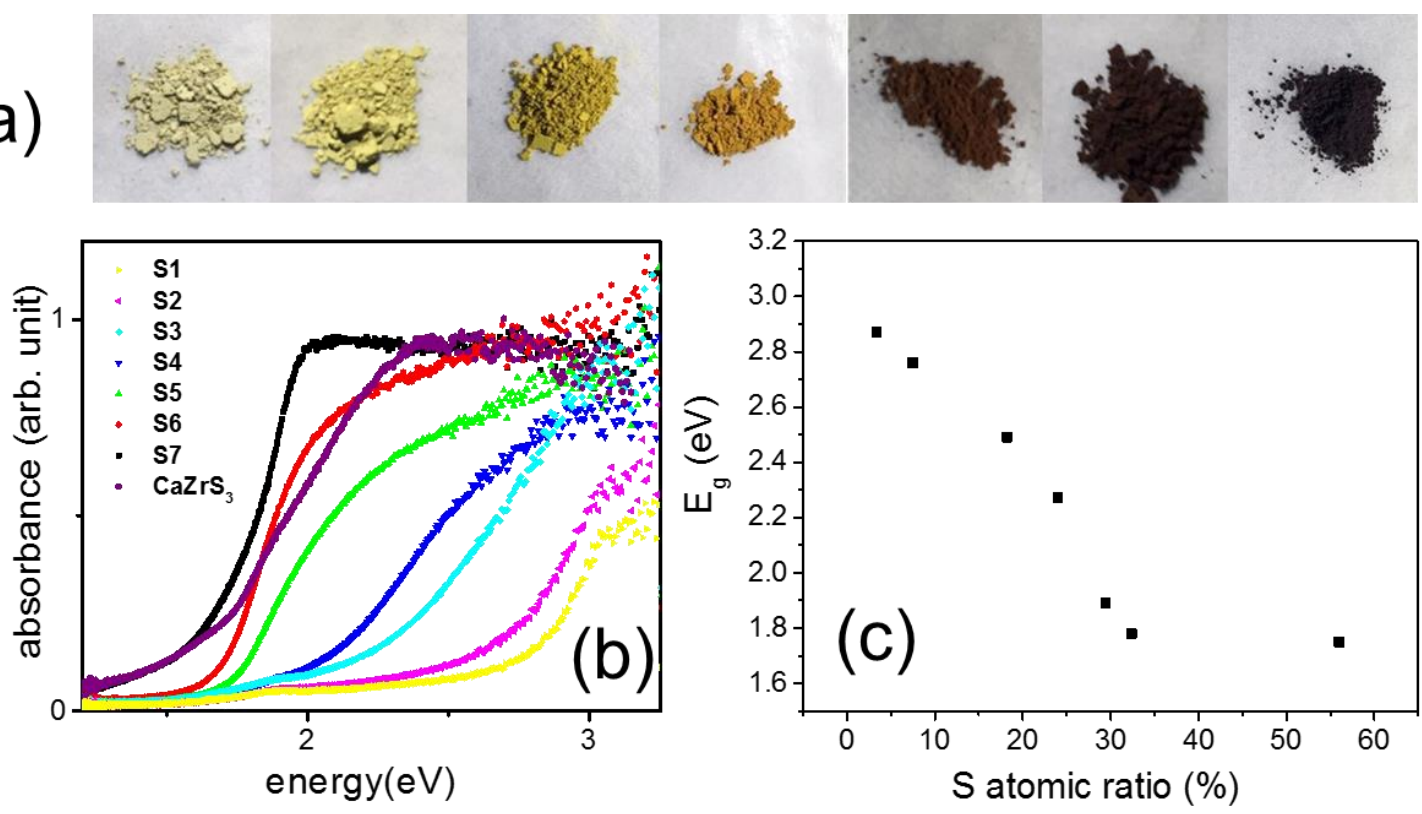

Figure 6 


\section{References}

[1] A. Kojima, K. Teshima, Y. Shirai, T. Miyasaka, J. Am. Chem. Soc., 131 (2009) 6050-6051.

[2] M. Liu, M.B. Johnston, H.J. Snaith, Nature, 501 (2013) 395.

[3] J. Burschka, N. Pellet, S.-J. Moon, R. Humphry-Baker, P. Gao, M.K. Nazeeruddin, M. Gratzel, Nature, 499 (2013) 316-319.

[4] J.-H. Im, C.-R. Lee, J.-W. Lee, S.-W. Park, N.-G. Park, Nanoscale, 3 (2011) 4088-4093.

[5] H.-S. Kim, C.-R. Lee, J.-H. Im, K.-B. Lee, T. Moehl, A. Marchioro, S.-J. Moon, R. Humphry-Baker, J.-H. Yum, J.E. Moser, M. Gratzel, N.-G. Park, Sci. Rep., 2 (2012) 591.

[6] H. Zhou, Q. Chen, G. Li, S. Luo, T.-b. Song, H.-S. Duan, Z. Hong, J. You, Y. Liu, Y. Yang, Science, 345 (2014) 542-546.

[7] C. Bi, Q. Wang, Y. Shao, Y. Yuan, Z. Xiao, J. Huang, Nat. Commun., 6 (2015) 7747.

[8] T. Zhang, M. Yang, Y. Zhao, K. Zhu, Nano Lett., 15 (2015) 3959-3963.

[9] http://www.nrel.gov/ncpv/

[10] G. Xing, N. Mathews, S. Sun, S.S. Lim, Y.M. Lam, M. Grtzel, S. Mhaisalkar, T.C. Sum, Science, 342 (2013) 344.

[11] S.D. Stranks, G.E. Eperon, G. Grancini, C. Menelaou, M.J.P. Alcocer, T. Leijtens, L.M. Herz, A. Petrozza, H.J. Snaith, Science, 342 (2013) 341-344.

[12] Q. Dong, Y. Fang, Y. Shao, P. Mulligan, J. Qiu, L. Cao, J. Huang, Science, 347 (2015) 967-970.

[13] W.J. Yin, T. Shi, Y. Yan, Appl. Phys. Lett., 104 (2014) 063903.

[14] M.L. Agiorgousis, Y.-Y. Sun, H. Zeng, S. Zhang, J. Am. Chem. Soc., 136 (2014) 14570-14575.

[15] M. Gratzel, Nat. Mater., 13 (2014) 838-842.

[16] M.D. McGehee, Nature, 501 (2013) 323-325.

[17] H. Hahn, U. Mutschke, Z. Anorg. Allg. Chem., 288 (1957) 269-278.

[18] A. Clearfield, Acta Crystallogr., 16 (1963) 135-142.

[19] R. Lelieveld, D.J.W. Ijdo, Acta Crystallogr., B36 (1980) 2223-2226.

[20] Y. Wang, N. Sato, T. Fujino, J. Alloy. Comp., 327 (2001) 104-112.

[21] C.-S. Lee, K.M. Kleinke, H. Kleinke, Solid State Sciences, 7 (2005) 1049-1054.

[22] M. Ishii, M. Saeki, Phys. Stat. Sol. (b), 170 (1992) K49.

[23] M. Ishii, M. Saeki, M. Sekita, Mater. Res. Bull., 28 (1993) 493-500.

[24] Y.-Y. Sun, M.L. Agiorgousis, P. Zhang, S. Zhang, Nano Lett., 15 (2014) 581-585.

[25] J.P. Perdew, A. Ruzsinszky, G.I. Csonka, O.A. Vydrov, G.E. Scuseria, L.A. Constantin, X. Zhou, K. Burke, Phys. Rev. Lett., 100 (2008) 136406.

[26] J. Heyd, G.E. Scuseria, M. Ernzerhof, J. Chem. Phys., 118 (2003) 8207-8215.

[27] P. Umari, A. Pasquarello, A. Dal Corso, Phys. Rev. B, 63 (2001) 094305. 
[28] M.N. Iliev, M.V. Abrashev, H.G. Lee, V.N. Popov, Y.Y. Sun, C. Thomsen, R.L. Meng, C.W. Chu, Phys. Rev. B, 57 (1998) 2872-2877.

[29] S. Venugopalan, M. Dutta, A.K. Ramdas, J.P. Remeika, Phys. Rev. B, 31 (1985) 1490-1497.

[30] N. Koshizuka, S. Ushioda, Phys. Rev. B, 22 (1980) 5394-5399.

[31] P.S. Dobal, A. Dixit, R.S. Katiyar, Z. Yu, R. Guo, A.S. Bhalla, J. Appl. Phys., 89 (2001) 8085-8091.

[32] Y. Zhang, B. Fluegel, M. C. Hanna, J. F. Geisz, L.-W. Wang, and A. Mascarenhas, phys. stat. sol. (b) 240 (2003) 396-403. 


\section{Vitae}

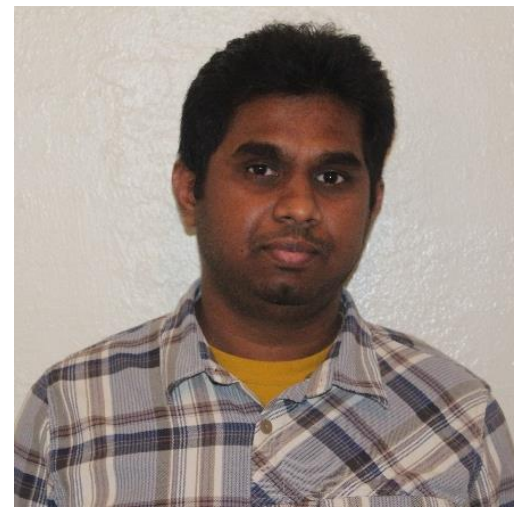

Samanthe Perera received his B. S. degree in Physics from the University of Colombo, Sri Lanka. He is currently a graduate student in the department of Physics at University at Buffalo. His research is focused on novel semiconducting materials for Photovoltaics.

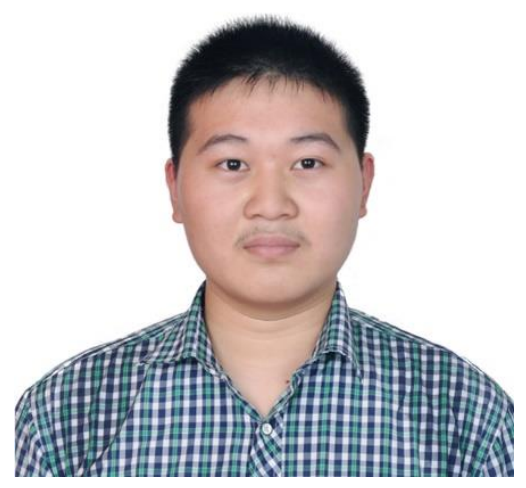

Haolei Hui is an undergraduate student from the Elite Class of School of Science, Xi'an Jiaotong University, China. He is presently an exchange student at the University at Buffalo, working on novel photovoltaic materials.

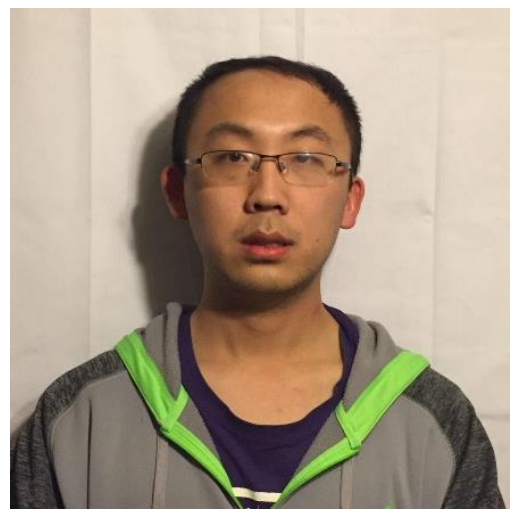

Chuan Zhao received his B. S. degree in Physics from the University of Science and Technology of China. He is currently a graduate student in the department of Physics at University at Buffalo. His current research interests are in two-dimensional semiconductors and materials for photovoltaics. 


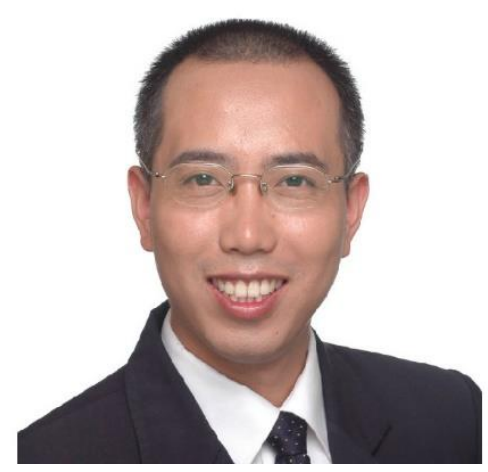

Hongtao Xue received his Ph.D. in Materials Science from the City University of Hong Kong in 2015. He is currently an associate professor of applied physics at Nanjing University of Posts and Telecommunications. His research interests focus mainly metal chalcogenide materials for electrochemical batteries, capacitors and photovoltaic cells.

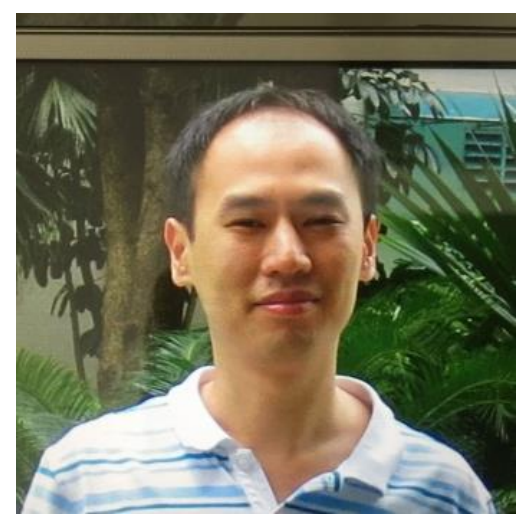

Fan Sun received his BS in physics at Nanjing University in 2009. He is currently a graduate student working towards his Ph.D, focusing on nanomagnetism and nanomaterial synthesis at SUNY at Buffalo.

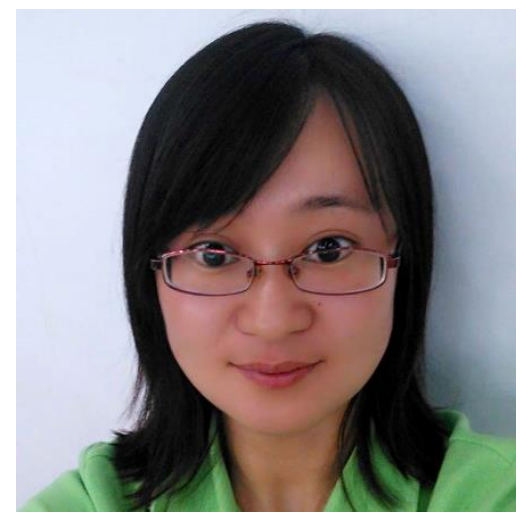

Chenhua Deng received her M. Sc. in Materials Science at Shanxi Normal University in 2012. She is currently a doctoral student working on her Ph.D. focusing on Chemistry and physics of nanoscale materials at Shanxi Normal University. 


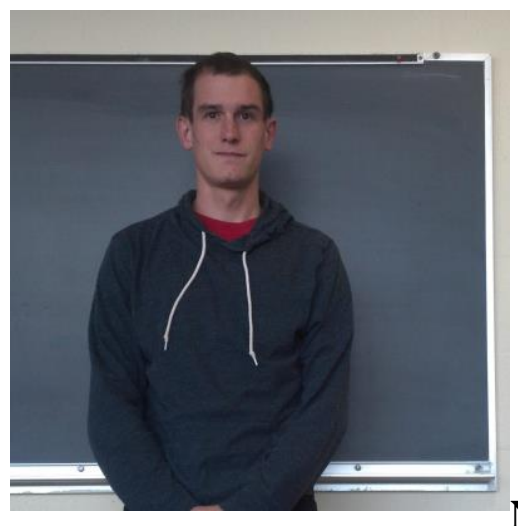

Nelson Gross received his BS in mathematics and physics at the College of Idaho in 2008. He is currently a graduate student working on his Ph.D. focusing on high pressure physics and optical properties of semiconductors at SUNY at Buffalo.

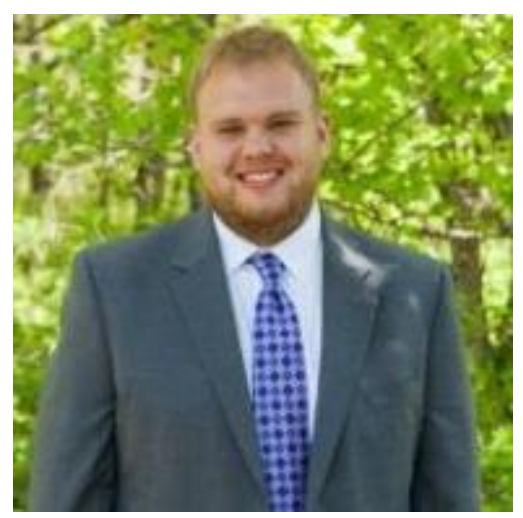

Christopher Milleville is a Ph.D. candidate under the supervision of Professor David F. Watson in the Department of Chemistry, University at Buffalo. His doctoral research focuses on colloidal quantum dot synthesis and surface functionalization to build nanoscale semiconductor heterostructures for solar energy conversion and storage.

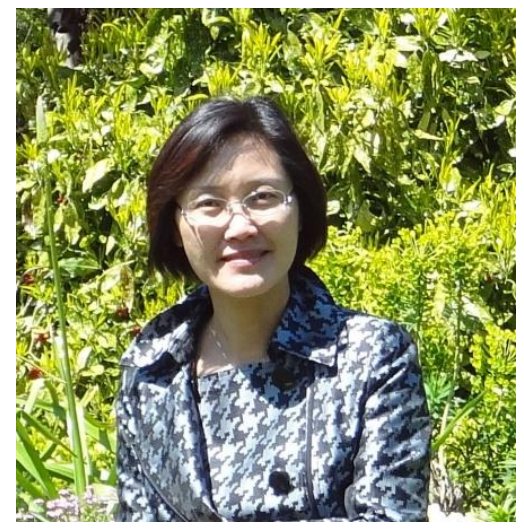

Xiaohong Xu received her Ph.D. in Material Science and Engineering from Xi'an Jiaotong University, China in 2001. From 2001 to 2006, she was in Huazhong University of Science and Technology, China, the University of Sheffield, UK, and Tohoku University, Japan as a postdoc or research fellow. In 2002, she became a Professor in Material Science at Shanxi Normal 
University. Her research includes oxide semiconductor spintronics, magnetic recording media, interface physics of heterostructures, two-dimensional materials and localized surface plasmon resonance. She is a Distinguished Young Scholar by the National Science Foundation of China.

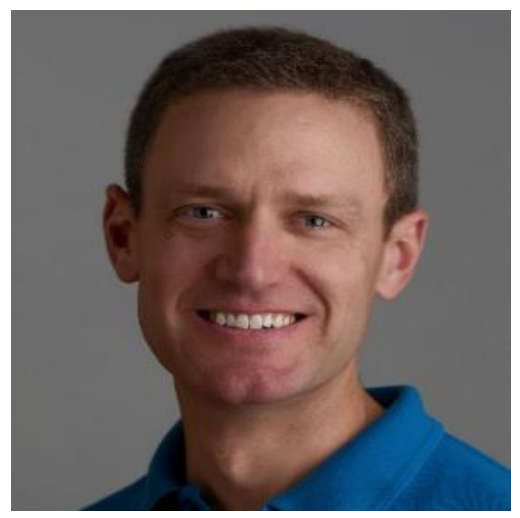

David Watson received his Ph.D. in Chemistry from Princeton University in 2001. He performed postdoctoral research at Johns Hopkins University then joined the Department of Chemistry at the University at Buffalo where he is now professor. His research interests are in nanostructured inorganic materials, surface chemistry, electron transfer processes, time-resolved spectroscopy, and photocatalysis.

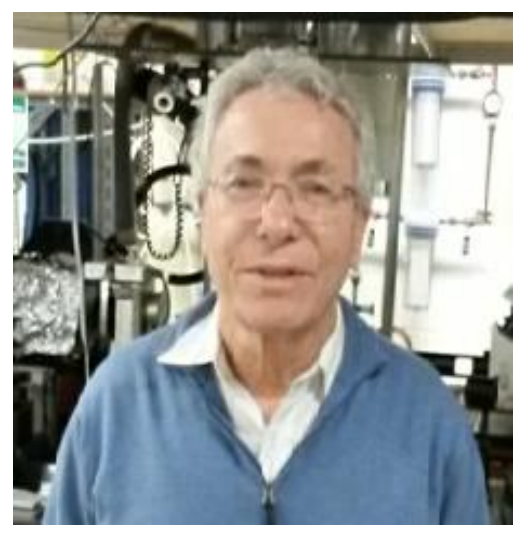

Bernard A. Weinstein received his Ph.D. in Physics from Brown in 1974, doing research under Manuel Cardona at the Max Planck Institute. He has held appointments as a postdoc at NIST, an assistant professor at Purdue, a Senior Scientist at Xerox WRC, and a visiting scientist at University Surrey, UK. In 1987 he was appointed a full professor in the SUNY at Buffalo Physics Department. His experimental research explores optical and high-pressure effects - with current investigations on phase changes and plastic deformation in semiconductors, photocrystallization in glasses, and properties of novel photovoltaic materials. He is an APS Fellow since 1997. 


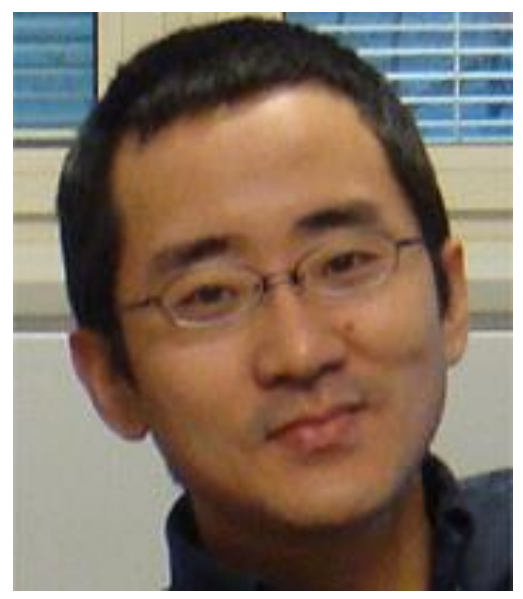

Yi-Yang Sun received his PhD degree from National University of Singapore (NUS) in 2004. Since then, he has worked as postdoc at NUS, National Renewable Energy Laboratory and Rensselaer Polytechnic Institute (RPI). In 2010, he was appointed Research Assistant Professor and later Research Scientist at RPI. His research interest is on the study of energy-related materials using first-principles computations. His current focus is on the design of novel solar cell materials, in particular, perovskite and other ionic semiconductors.

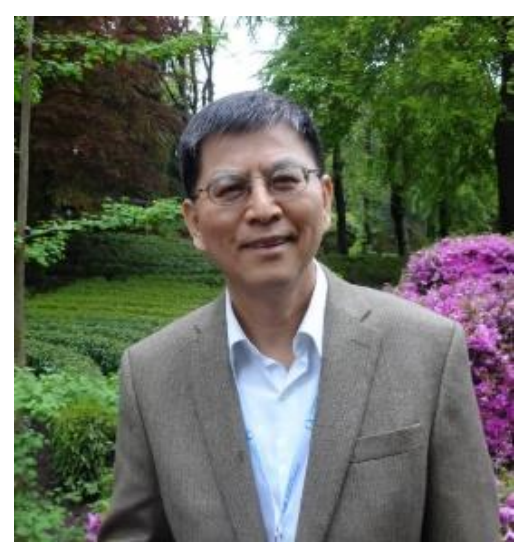

Shengbai Zhang received his Ph.D. in Physics from the University of California at Berkeley in 1989. He moved to Xerox PARC as a postdoc, before joining the National Renewable Energy Laboratory in 1991. In 2008, he became the Senior Kodosky Constellation Chair and Professor in Physics at Rensselaer Polytechnic Institute. His computational research covers a wide range of materials for bulk properties, defect structures, and surface physics. His recent work involves emerging low-cost photovoltaic materials, phase change memory materials, topological insulators, Weyl semimetals, two-dimensional layered materials, and excited state dynamics. $\mathrm{He}$ is a Fellow of the American Physical Society since 2001. 


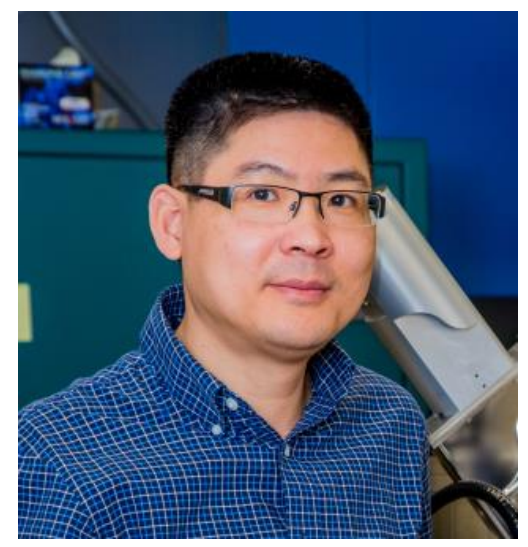

Hao Zeng received his Ph.D. in physics from the University of Nebraska-Lincoln in 2001. He joined the Department of Physics at the University at Buffalo in 2004 after a 3-year postdoc at IBM T.J. Watson Research Center, and was promoted to full professor in 2014. His research interests are in nanoscale magnetism and magnetic materials, spintronics, and photovoltaic materials and devices. 
Table 1. Assignment of experimental Raman peaks according to the irreducible representations of point group $D_{2 \mathrm{~h}}$ based on calculated frequencies. The unit is $\mathrm{cm}^{-1}$.

\begin{tabular}{|l|l|l|l|l|l|l|l|l|}
\hline mode & Theory & Exp. & mode & Theory & Exp. & mode & Theory & Exp. \\
\hline$B_{3 \mathrm{~g}}{ }^{5}$ & 418.1 & -- & $B_{3 \mathrm{~g}}{ }^{3}$ & 203.0 & 207 & $A_{\mathrm{g}}{ }^{3}$ & 98.8 & 97 \\
\hline$B_{2 \mathrm{~g}}{ }^{7}$ & 399.1 & -- & $B_{2 \mathrm{~g}}{ }^{5}$ & 201.9 & 207 & $B_{2 \mathrm{~g}}{ }^{3}$ & 97.6 & 93 \\
\hline$B_{1 \mathrm{~g}}{ }^{5}$ & 388.0 & 392 & $B_{1 \mathrm{~g}}{ }^{3}$ & 174.5 & -- & $\mathrm{B}_{1 \mathrm{~g}}{ }^{1}$ & 85.8 & 83 \\
\hline$A_{\mathrm{g}}{ }^{7}$ & 304.6 & 304 & $A_{\mathrm{g}}{ }^{5}$ & 168.7 & 169 & $B_{2 \mathrm{~g}}{ }^{2}$ & 81.3 & 78 \\
\hline$B_{1 \mathrm{~g}}{ }^{4}$ & 290.8 & -- & $B_{2 \mathrm{~g}}{ }^{4}$ & 167.8 & 169 & $B_{3 \mathrm{~g}}{ }^{1}$ & 76.9 & 72 \\
\hline$B_{3 \mathrm{~g}}{ }^{4}$ & 290.3 & -- & $B_{3 \mathrm{~g}}{ }^{2}$ & 160.2 & -- & $A_{\mathrm{g}}{ }^{2}$ & 76.0 & 72 \\
\hline$B_{2 \mathrm{~g}}{ }^{6}$ & 214.8 & 224 & $A_{\mathrm{g}}{ }^{4}$ & 142.7 & 143 & $B_{2 \mathrm{~g}}{ }^{1}$ & 64.0 & 64 \\
\hline$A_{\mathrm{g}}{ }^{6}$ & 207.0 & 216 & $B_{1 \mathrm{~g}}{ }^{2}$ & 111.0 & -- & $A_{\mathrm{g}}{ }^{1}$ & 58.7 & 57 \\
\hline
\end{tabular}


Table 2. The atomic percentage of the 4 elements ( $\mathrm{Ba}, \mathrm{Zr}, \mathrm{O}$ and $\mathrm{S}$ ) for samples $\mathrm{S} 1$ to $\mathrm{S} 7$, as measured by EDX spectroscopy.

\begin{tabular}{|c|r|r|r|r|r|r|r|}
\hline & \multicolumn{1}{|c|}{ S1 } & \multicolumn{1}{c|}{ S2 } & \multicolumn{1}{c|}{ S3 } & \multicolumn{1}{c|}{ S4 } & \multicolumn{1}{c|}{ S5 } & \multicolumn{1}{c|}{ S6 } & S7 \\
\hline $\mathbf{B a}$ & 25.08 & 25.09 & 23.42 & 21.98 & 25.54 & 25.64 & 23.79 \\
\hline $\mathbf{Z r}$ & 20.68 & 20.9 & 21.26 & 20.31 & 21.48 & 22.23 & 22.31 \\
\hline $\mathbf{O}$ & 50.85 & 46.55 & 37.1 & 33.68 & 23.59 & 19.74 & 0 \\
\hline $\mathbf{S}$ & 3.38 & 7.46 & 18.22 & 24.03 & 29.4 & 32.4 & 53.89 \\
\hline
\end{tabular}

